\title{
Localized Power Control for Multihop Large-Scale Internet of Things
}

\author{
Ahmed Bader, Senior Member, IEEE and Mohamed-Slim Alouini, Fellow, IEEE
}

\begin{abstract}
In this paper, we promote the use of multihop networking in the context of large-scale Internet of Things (IoT). Recognizing concerns related to the scalability of classical multihop routing and medium access techniques, we advocate the use of blind cooperation in conjunction with multihop communications. However, we show that blind cooperation is actually inefficient unless power control is applied. Inefficiency in this paper is projected in terms of the transport rate normalized to energy consumption. To that end, we propose an uncoordinated power control mechanism whereby each device in a blind cooperative cluster randomly adjusts its transmit power level. We derive an upper bound on the mean transmit power that must be observed at each device. We also devise a practical mechanism for each device to infer about the size of its neighborhood; a requirement necessary for the operation of the power control scheme. Finally, we assess the performance of the developed power control mechanism and demonstrate how it consistently outperforms the point-to-point case.
\end{abstract}

\section{INTRODUCTION}

\section{A. Motivation}

The Internet-of-Things (IoT) is emerging as one of the major trends shaping the development of technologies in the Information and Communications Technology (ICT) sector at large [1]. The Internet paradigm is being reshaped to encompass smart objects in addition to human end-devices [2]. As such, the IoT is clearly providing a convergence space for many applications verticals [3], particularly wireless sensor networks (WSN) and machine-to-machine (M2M) communication networks.

The IoT is expected to be large in scale along multiple dimensions particularly in terms of span as well as density and number of interconnected devices [2]. A recent report from ABI Research predicts that $75 \%$ of the growth in wireless connections between today and the end of the decade will come from non-hub devices: sensor nodes and accessories [4]. A research report from International Data Corporation (IDC) expects the installed base of the IoT to be approximately 212 billion "things" globally by the end of 2020 [5]. As such, a large-scale IoT indeed mandates scalable communications and networking protocols. Yet, it is one that is sensitive to form factor and energy consumption [6]. Since we perceive WSN and M2M applications as precursors to the IoT [7], it is then inevitable to apply what we have learned there in terms of

Manuscript received XXX, revised XXX. The authors are with King Abdullah University of Science and Technology (KAUST). Emails: \{ahmed.bader,slim.alouini\}@kaust.edu.sa. This work was funded under grant AT-34-185 from King Abdulaziz City of Science and Technology (KACST). This is an expanded version of work submitted for presentation in the IEEE Gloecom'2015 Workshop on Optimizing Heterogeneous Networking Technologies for the Internet of Things, San Diego, CA. scalability under energy constraints.

To that end, multihop networking has been often advocated as a viable tool meeting the design objectives. Examples include smart utility/grid [8] and vehicle-to-vehicle networks [9] whereby multihop networking has been incorporated into a standardized protocol stack. As a matter of fact, an industryleading work-group, THREAD, has recently released a wireless mesh (multihop) networking protocol tailored for the IoT of the future [10].

However, the extension of multihop networking protocols for use in large-scale IoT seems to be a debatable issue. Some researchers have voiced concerns that the IoT setup pushes the envelope of scalability to an extent that discourages the use of multihop networking [2]. This may have been motivated by the fact that the routing and medium access protocols overhead builds up quite rapidly as the network grows in scale [11]. This is particularly true for dynamic networks whose topologies undergo frequent changes due to mobility and/or uncoordinated sleep schedules [12]. Indeed, the utilization of multihop networking for large-scale IoT must first address the concerns raised above. In specific, for the network to scale we need to tackle the challenge of protocol overhead [13].

Undoubtedly, there could exist a few approaches at various protocol layers to address that issue. One direction which we believe is quite promising, yet still poorly investigated is the use of "blind cooperative relaying". In [14], blind cooperative relaying was formally introduced for the first time. The relaying mechanism was actually dubbed there as "randomized distributed cooperative transmission". In essence, blind cooperative relaying entails the forwarding of packets while not reverting to any relay selection or election process.

The term blind mainly stems from the fact that devices within a cooperative cluster are actually unaware of each other [15]. This in itself is quite valuable from the perspective of protocol overhead minimization. As a bonus, blind cooperative relaying also offers two types of link gains: array (power) and diversity gains [16]. The value of blind cooperation becomes clear when contrasted to classical cooperative techniques. The latter typically require cooperative devices to align or coordinate their transmit space-time coding (STC) matrices. Indeed, such coordination entails a substantial number of messaging and transactions between cooperative devices. Consequently, the resulting overhead can not be ignored and only grows with increasing device density. Blind cooperation instead calls for randomization of STC matrices at each device. The result is an array gain in the order of $\mathcal{O}(N)$, where $N$ is the number of cooperative devices. The diversity gain surely depends on the underlying precoding STC matrix structure. Broadly speaking, a diversity gain of at least $3 \mathrm{~dB}$ is attained, whenever $N \geq 2$ 
[14].

Despite being a promising area of research, the study of blind cooperative relaying in the context of large-scale networks, i.e. IoT, has not picked up pace yet. Albeit some followup work on asynchronous transmissions [17] and distributed medium access [18], the performance of blind cooperative relaying is yet to be properly addressed. One of the key performance metrics to consider for large-scale IoT is the transport rate. This is defined as the "average number of bits that can be communicated over distance per unit time" [19].

The seminality of the transport rate as a performance metric has been identified early on in [19] and others followed through (see for example [20], [21]). In an IoT setup however, energy is definitely a precious commodity. Hence, in this paper we normalize it by the aggregate energy consumption. Having said that, it is paramount when employing blind cooperative relaying to have some means to control the aggregate transmit power. In other words, there must be a power control mechanism in place. Otherwise, the performance in terms of normalized transport rate (NTR) will be obviously very poor. As a matter of fact, we show in this paper that the NTR in case of point-to-point transmission is generally higher than the case of blind cooperative transmission without power control.

Based on the above, the motivation for devising transmit power control becomes evident. Any mechanism to be devised has to be localized, i.e. each device within a cluster locally decides on an appropriate power level. Clearly, there is no room here for cross-device coordination or exchange of information as this will defy the original virtue of coordination-free relaying.

\section{B. Approach}

To address the power control requirement, a viable mechanism must be developed; which is the core contribution of this work. Such a mechanism features uncoordinated localized power control at each device within a cooperative cluster. For $N$ cooperative devices the transport rate $T$ is given by:

$$
T=\frac{R}{\sum_{n=1}^{N} P_{n}}, P_{n} \leq P_{\max }
$$

where $R$ is the maximum communication range, and $P_{n}$ is the transmit power of the $n$th cooperative device under a peak power constraint of $P_{\max }$. This is true assuming fixed frame duration and link rate, hence they are dropped in the subsequent analysis for convenience.

In this work, we do not treat this power control problem from an optimization point of view. In other words, we do not simply seek to maximize the NTR over transmit power distributions (like for instance the approach adopted in [22], [23]). While such an approach is quite valid and intriguing when dealing with throughput as a performance metric, it is not quite the case from the perspective of NTR. As a matter of fact, attempting to maximize NTR only over transmit power leads to trivial solutions. This is best argued by means of the following example. One such problem formulation is to solve for $\arg \max _{\left\{P_{n}\right\}_{n=1}^{N}} T$ s.t. $R \geq R_{1}$, where $R_{1}$ could be the expected distance to the closest neighbor [22]. The optimization constraint on $R$ is equivalent to placing a lower bound on $\sum_{n=1}^{N} P_{n}$. We will show in the next section that NTR is a negative monotone in $\sum_{n=1}^{N} P_{n}$. Consequently, the problem $\arg \max _{\left\{P_{n}\right\}_{n=1}^{N}} T$ s.t. $R \geq R_{1}$ is simply solved by placing $\sum_{n=1}^{N} P_{n}$ at its lower bound.

Our approach in this paper is rather to benchmark the power control mechanism we develop against the elementary case of point-to-point transmission, that is the case of $N=1$. To state our goal in this paper in more explicit terms: it is to devise a power control mechanism such that the NTR of blind cooperative transmission is higher (in the average sense) than the simple case of point-to-point transmission.

\section{Contributions}

Uncoordinated power control has been studied before in literature [22], [23], [24], [25]. Nonetheless, our work here is differentiated in the following manner:

1) Performance in [24], [25] is evaluated only in terms of outage probability. In [22], [23] the outage probability analysis is taken further to evaluate throughput performance. In our work, we rather consider the normalized transport rate (NTR). The rationale behind the choice of NTR as a key performance metric is intimately related to the large-scale nature of a multihop IoT. In contrast to the throughput metric, NTR also captures hop distance (which eventually affects end-to-end delay) as well as energy consumption.

2) More specifically, the work in [23] studies optimal power control strategies over point-to-point links for noiselimited scenarios. The fundamental assumption is that a single transmission can span multiple trials before success. In the context of large-scale blind cooperative relaying, this is indeed a luxury that we cannot afford.

3) It is assumed in [22], [23], [24], [25] that the receiver or at least the distance to the receiver is already known to the transmitter. More specifically, it is assumed in [22] that the link is established to the nearest neighbor. This does not lend itself to effective blind cooperation since cooperative devices neither have knowledge of the cooperative cluster members nor about the receiver(s). Moreover, routing to the nearest neighbor [22] seems to be impractical taking into consideration that routing protocols in large-scale are generally designed to maximize the link distance (i.e. transmit to the furthest neighbor) [26].

4) In [22], the criterion for a successful link is represented using the outage probability. This in itself does not account for link availability. To reflect the link availability onto the success criterion, we condition the outage probability to be larger than a minimum acceptable availability threshold.

5) Other works attempt to devise power control strategies for a set of distinct packet transmissions. Here, we rather focus on power control within a cluster of devices concurrently engaged in the same packet transmission. We actually see these two lines of work as complementary or tangential to each other. 
Another major contribution of this paper is the development of an online learning mechanism for the sake of estimating the size of the cooperative cluster. It is a two-step process based on an orthogonal frequency division multiplexing (OFDM) physical (PHY) layer. The mechanism enables each cooperative device to locally scale its own transmit power based on an estimate of the number of neighboring cooperative devices. We also show that typical IoT deployment modes drive the network to be noise-limited due to penetration losses. We develop a structured methodology to qualify whether a specific IoT setup tends to be noise-limited or not.

The rest of the paper is organized as follows. Section II offers an extensive discussion of the link and network models. It also provides an analytical and numerical evidence for adopting a noise-limited scenario for the IoT. The problem formulation is also developed in detail in the same section. In Section III the online learning process is developed. The performance of the power control mechanism is assessed in Section IV and then paper is concluded in Section V.

\section{Problem Setup}

\section{A. Network Model}

We assume that the source $S$ is scheduled to send $y=$ $1 \ldots Y$ consecutive frames to destination $D$ in a multihop fashion, over a network of stationary or moderately mobile IoT devices. The transmission of an ensemble of $Y$ frames may correspond to frame fragmentation imposed by higher-layer convergence protocols such as 6LoWPAN [30]. An IoT device is assumed to have the localized ability to distinguish whether it makes positive progress towards the sink or not. In one of our previous works we have devised two different mechanisms to accomplish that mission [31]. Hence, relaying and routing in the context of this paper become two interchangeable terms.

Each cluster is assumed to be confined to an annular sector with angle $\theta$ and radii $v_{1}, v_{2}, v_{1}<v_{2}$ [32], [33]. The origin of the annular sector lies at the receiver (Fig. 1 in [33]). It was shown in [33] that a cluster of $N$ devices can be equivalently represented by one virtual device located at a radial distance of $v_{1}$, and whose transmit power is $\phi=K \sum_{n=1}^{N} P_{n}$, where $P_{n}$ is the transmit power of node $n$. Here, $K$ is a scaling factor that absorbs the spatial distribution of cooperative devices and is derived in the next subsection.

Generally, there exists $I+1$ concurrent transmissions (each carrying a distinct packet) on the same radio channel. Each packet transmission $i \in\{0, \ldots I\}$ is carried out by a cluster of $N_{i}$ blind cooperative devices. The wanted transmission is that designated by $i=0$. The equivalent transmit power of a cooperative cluster is denoted by $\phi_{i}, i=0 \ldots I$.

\section{B. Link model}

The communication range $R$ for the wanted transmission is dictated by the outage probability, link availability criterion $\tau$, target detection threshold $\gamma_{t}$, the diversity gain $\gamma_{d}$, and the noise floor $W$. The diversity gain depends upon the underlying randomized cooperation scheme. In [14], it was shown that with Alamouti coding, $\gamma_{d}=0 \mathrm{~dB}$ for $N=1$, and $\gamma_{d}=3$ $\mathrm{dB}$ for $N \geq 2$. The received signal strength is denoted by $s_{i}, i=0 \ldots I$. The distance between the $i$ th interferer and the receiver of the wanted transmission is denoted by $D_{i}$.

Block Rayleigh fading is assumed such that $\left\{s_{i}\right\}_{i=1}^{I}$ are independent exponentially with probability density function (pdf) $f_{s_{i}}\left(s_{i}\right)=e^{s_{i} / \bar{s}_{i}} / \bar{s}_{i}, s_{i}>0$. Nonetheless, IoT devices are assumed to suffer from various penetration losses. Denoting the probability of suffering from penetration loss $l$ as $f_{l}(l)$, then the average received power is expressed as

$$
\overline{s_{i}}=\phi_{i} \bar{l}\left(\frac{\lambda}{4 \pi x}\right)^{\alpha}, x \in\left\{R, D_{i}\right\}
$$

where $\bar{l}=\sum_{l} l f_{l}(l)$. An IoT device may be placed virtually anywhere: deep indoors inside a building or on its facade, in a vehicle or on top of it, outdoors on a sidewalk or a street, ... etc. This is a direct corollary of the IoT hosting a multitude of device types such as a smart utility meter, a smart grid sensor, a smart object or sensor in a vehicle, a humanwearable device, and so on. The IoT device in each case will suffer from a different penetration loss. It will be shown later in this section that taking into consideration the disparity of penetration losses is paramount to determine whether an IoT scenario is interference- or noise-limited.

The successful link condition is derived in [34]. Accounting for a link availability threshold $\tau$, a successful link is established if

$$
\tau e^{\gamma_{t} W / \gamma_{d} \overline{s_{o}}} \prod_{i=1}^{I}\left(1+\frac{\gamma_{t} \overline{s_{i}}}{\gamma_{d} \overline{s_{o}}}\right) \geq 1
$$

If the interference distances $\left\{D_{i}\right\}_{i=1}^{I}$ are given, then the communication range $R$ is computed by substituting (2) into (3). It is worthwhile mentioning that $D_{i}$ is measured to the location of the virtual point representing the $i$ th interfering cooperative cluster.

For noise-limited scenarios, the link condition in (3) reduces to $\tau e^{\gamma_{t} W / \gamma_{d} \overline{s_{o}}} \geq 1$. Under such circumstances the from (2) we obtain:

$$
R=\frac{\lambda}{4 \pi}\left(\frac{-\gamma_{d} \phi_{o} \bar{l} \ln \tau}{W \gamma_{t}}\right)^{1 / \alpha}
$$

\section{Noise-limited IoT scenarios}

We define an IoT application scenario to be noise-limited if

$$
\mathbb{P}\left[\sum_{i=1}^{I} s_{i}<W\right]>\eta
$$

where $0<\eta<1$. In other words, if $\eta$ is large enough, then an IoT deployment scenario can be comfortably described to be noise-limited.

One can argue that the condition in (5) is not sufficient in practice to qualify that a scenario is noise-limited. This is true since very small communication ranges will still satisfy the condition. To make the noise-limited condition in (5) more meaningful we place a lower bound on the interference-free communication range given by (4) such that $R \geq R_{o}$.

We define $S_{I}=\sum_{i} s_{i}$. It can be shown then that the probability density function $f_{S_{I}}\left(S_{I}\right)$ is given by [35]

$$
f_{S_{I}}\left(S_{I}\right)=\sum_{i=1}^{I} a_{i} f_{s_{i}}\left(S_{I}\right), a_{i}=\prod_{j=1, j \neq i}^{I} \frac{1}{1-\overline{s_{j}} / \overline{s_{i}}}
$$


TABLE I

NOTATIONS AND TYPICAL VALUES

\begin{tabular}{|c|c|c|c|}
\hline$I$ & number of concurrent packet transmissions & $N_{i}$ & number of coop. nodes transmitting packet $i$ \\
\hline$P_{\max }$ & peak transmit power, $[10-20] \mathrm{dBm}$ & $P_{n}$ & transmit power of node $n$ \\
\hline & comm. range of wanted transmission & $D_{i}$ & interference distance, $i=1 \ldots I$ \\
\hline$s_{i}$ & received signal power & $\lambda$ & wavelength \\
\hline$\alpha$ & path-loss exponent, $\alpha \in[2.7-3.5]$ & $\phi_{i}$ & equivalent transmit power of coop cluster $i$ \\
\hline$\gamma_{d}$ & transmit diversity gain, $\gamma_{d}=3 \mathrm{~dB}$ when $N_{0} \geq 2$ & & link availability threshold, $\tau=90 \%$ \\
\hline$A$ & area confining cluster of cooperative devices & $\begin{array}{l}\theta \\
v_{1}, v_{2}\end{array}$ & $\begin{array}{l}\text { angle of the annular sector confining coop. devices } \\
\text { inner and outer radii of the annular sector }\end{array}$ \\
\hline$T$ & normalized transport rate & & noise-limited scenario threshold, $\eta=80 \%$ \\
\hline$\rho$ & average spatial node density & & \\
\hline$W$ & $\begin{array}{l}\text { noise power assuming noise floor of } 10 \mathrm{~dB} \\
\text { and occupied bandwidth of } 2.7 \mathrm{MHz} \\
\text { (similar to IEEE 802.15.4-based systems [27]) }\end{array}$ & & $\begin{array}{l}\text { desired SINR threshold, } \gamma_{t}=3 \mathrm{~dB} \text { for BPSK } \frac{1}{2} \\
\text { penetration loss, } l=7,15,20 \mathrm{~dB} \text { for } \\
\text { vehicle, in-building, and deep indoor [28], [29] }\end{array}$ \\
\hline$c$ & hop number & $y$ & frame index, $y=1 \ldots Y$ \\
\hline B & feedback set of subcarriers, $|\mathbf{B}|=M_{B}=32$ & $\mathbf{F}$ & feed-forward set of subcarriers, $|\mathbf{F}|=M_{F}=96$ \\
\hline$\Omega_{c}$ & set of coop. devices at hop $c$ & $K$ & spatial absorbtion factor in virtual node representation \\
\hline
\end{tabular}

Subsequently, the cumulative density function is given by:

$$
\begin{aligned}
F_{S_{I}}\left(S_{I}\right) & =\sum_{i=1}^{I} \frac{a_{i}}{\overline{s_{i}}} \int_{0}^{S_{I}} e^{-S_{I} / \overline{s_{i}}} d S_{I} \\
& =\sum_{i=1}^{I} a_{i}\left(1-e^{-S_{I} / \overline{s_{i}}}\right)
\end{aligned}
$$

It follows that a scenario is qualified as being noise-limited if the following condition is satisfied:

$$
\sum_{i=1}^{I} a_{i}\left(1-e^{-W / \overline{s_{i}}}\right)>\eta, \quad \text { and } \quad R \geq R_{o}
$$

To put things into context, we have evaluated the noiselimited condition of (8) for what we believe is a typical IoT deployment scenario. We have selected $R_{o}=30$ meters, which is the typical separation between two neighboring residential or mid-size buildings. We also know from [36] that the path loss exponent $\alpha$ has a profound impact on the wireless link. As such, we run the computation for $\alpha \in[2.7-3.5]$ which is a typical range for urban environments [37]. Taking $I=4$, $\eta=80 \%$, and $\left(\max D_{i}-\min D_{i}\right) \leq 50$ meters, we search for realizations of $\left\{D_{i}\right\}_{i=1}^{I}$ satisfying (8) for the given value of $\alpha$. We also consider $P_{\max } \in[10-20] \mathrm{dBm}$. The rest of the parameter values are provided in Table I.

Results are provided in Figure 1. Two scenarios have been studied depending on the breakdown of penetration losses. The first one features a larger inclination towards outdoor deployment of IoT devices, and thus is expected to be more sensitive to co-channel interference. The second scenario features a uniform distribution of devices over all four modes: outdoors, in-vehicle, in-building, and deep indoors. As evident from Figure 1, the average interferer's distance driving an IoT setup to be noise-limited can be as low as 120 meters for large values of the path loss exponent, $\alpha$. For smaller values of $\alpha$ and under dominant outdoor deployment (scenario 2), the average interference distance is no more than 220 meters. The results above strongly suggest that IoT may be comfortably regarded as being noise-limited.

To further back up such an argument, we also benchmarked the results obtained above against the spatial (area) throughput

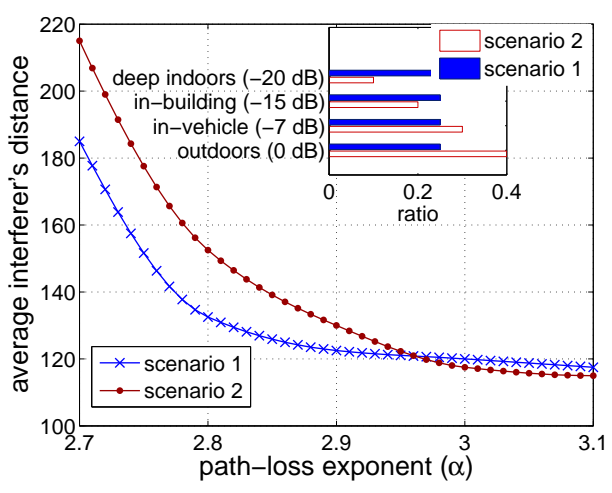

Fig. 1. The average interference distance such that a setup may be considered to be noise-limited is plotted here against a range of path loss exponent values. Two scenarios are featured. Scenario 1: IoT devices mainly deployed outdoors, e.g. sidewalks, streets, parks, light poles. Scenario 2: equal split of deployment modes to account for smart building objects deployed indoors and vehicular IoT devices.

density expected from an IoT. Although there does not exist yet empirical data to consult with, but we can draw parallels from the world of smart utility networks (SUN). A few SUN usage and traffic patterns were reported in [38]. One of the most traffic-intense patterns is the Adaptive Metering Infrastructure (AMI) with a typical message size of $10 \mathrm{kbits}$ and an update frequency of $5 \mathrm{~s}^{-1}$. Assuming a building density of $900 \mathrm{~m}^{-2}$, and 10 smart meters per building, then the total throughput density is $22.2 \mathrm{Mbps} / \mathrm{km}^{-2}$. The minimum throughput density featured in Figure 1 corresponds to $I=5$, link rate of $1.2 \mathrm{Mbps}$, and $\overline{D_{i}}=217 \mathrm{~m}$, which yields 40 $\mathrm{Mbps} / \mathrm{km}^{-2}$, hence reinforces the conclusion that IoT setups may be generally considered to be noise-limited.

\section{Virtual Node}

The radial distance $R_{n}$ away from a receiver has a pdf (see Eq. 12 in [39]):

$$
f_{R_{n}}\left(R_{n}\right)=\frac{2 R_{n}}{v_{2}^{2}-v_{1}^{2}}, R_{n} \in\left[v_{1}, v_{2}\right]
$$




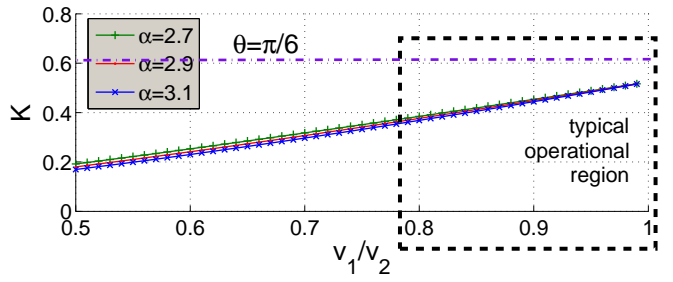

Fig. 2. The spatial absorption factor $K$ as function of the ratio of the annular sector radii $v_{1}$ and $v_{2}$.

where we recall that $v_{1}, v_{2}$ are the radii of the annular sector confining the cooperative devices. The angle of each node $n$ within the cluster is also assumed to be uniformly distributed over the range $\left[-\frac{\theta}{2}, \frac{\theta}{2}\right]$. As such we have

$$
\mathbb{E}_{R_{n}}\left[\frac{1}{R_{n}}\right]=\frac{2 \theta}{2-\alpha}\left(\frac{v_{2}^{2-\alpha}-v_{1}^{2-\alpha}}{v_{2}^{2}-v_{1}^{2}}\right)
$$

From (2), we can express

$$
\overline{s_{o}}=\left(\frac{\lambda}{4 \pi v_{1}}\right)^{\alpha} K \sum_{n=1}^{N} P_{n}
$$

Therefore, we obtain an expression for the spatial absorbtion factor $K$ :

$$
K=v_{1}{ }^{\alpha} \mathbb{E}_{R_{n}}\left[\frac{1}{R_{n}}\right]=\frac{2 \theta}{2-\alpha}\left(\frac{v_{1}}{v_{2}}\right)^{\alpha}\left(\frac{1-\left(v_{1} / v_{2}\right)^{2-\alpha}}{1-\left(v_{1} / v_{2}\right)^{2}}\right)
$$

It can be straightforwardly shown that $\lim _{v_{2} \rightarrow v_{1}} K=\theta$. Moreover, assuming a typical operational range of $v_{1} / v_{2} \in$ $[0.8,1)$, then Figure 2 numerically shows that $K \approx \theta$ is an acceptable approximation. This is true independent of the path loss exponent $\alpha$ as shown in the figure.

\section{E. Problem Formulation}

So far, we have established the conclusion that the IoT is mostly noise-limited. Subsequently, we can now focus the power control mechanism within the confines of one cooperative cluster; that of the wanted packet transmission. As such, in the subsequent analysis we will drop the index corresponding to the wanted transmission. We recall that blind cooperation eliminates the need for coordination among cooperative devices. Consequently, a device within the cooperative cluster is unaware of $N$, i.e. the number of cooperative devices. A device will have to locally set its transmit power level, $P_{n}$.

Now to further motivate the need for power control, we address the following question: what if all devices within a cluster transmit at $P_{n}=P_{\max }$ such that $\phi=K N P_{\max }$ ? We are about to demonstrate that this will render cooperation less efficient compared to the simple point-to-point case, i.e. with $N=1$.

From (4) and (1), we can express the ratio of NTR between both cases as:

$$
\frac{T_{(N \geq 2)}}{T_{(N=1)}}=(2 \theta)^{1 / \alpha}\left(\frac{\sum_{n=1}^{N} P_{n}}{P_{\max }}\right)^{\frac{1-\alpha}{\alpha}}
$$

Our goal is for blind cooperation to enjoy better NTR compared to the point-to-point case in the average sense, i.e. $\mathbb{E}\left[T_{(N \geq 2)} / T_{(N=1)}\right] \geq 1$. Since $T_{(N \geq 2)} / T_{(N=1)}$ is convex, then by means of Jensen's inequality as, our target becomes to satisfy:

$$
(2 \theta)^{1 / \alpha}\left(\frac{\bar{P}_{n} \bar{N}}{P_{\max }}\right)^{\frac{1-\alpha}{\alpha}} \geq 1
$$

The degree of convexity in $T_{(N \geq 2)} / T_{(N=1)}$ increases as $\bar{N}$ increases. This is due to the fact that $\sum_{n=1}^{N} P_{n}$ is a positive monotone in $N$. Thus, the Jensen's inequality use in (14) becomes less tight and subsequently less accurate with increasing $\bar{N}$. To compensate for that, we introduce a scaling coefficient $\beta$ which is a negative monotone in $\bar{N}$ such that (14) is adjusted to

$$
\overline{P_{n}} \leq \frac{P_{\max }(2 \theta)^{\frac{1}{\alpha-1}}}{\beta \bar{N}}
$$

Without power control, $P_{n}=P_{\max }, \forall n$. As such, the condition in (15) is satisfied for $\bar{N} \leq(2 \theta)^{\frac{1}{\alpha-1}}$. However, we can easily shown by means of a numerical example that it is quite challenging to meet this condition. Practically, we expect $\theta$ to belong to an interval ${ }^{1}$ centered around $\frac{\pi}{4}$. Subsequently, we need to have $\bar{N} \in[1.2,1.3]$. This is indeed highly unlikely in a large-scale dense IoT setup. For instance if $v_{1}=50 \mathrm{~m}$ and $v_{2}=75 \mathrm{~m}$, then $\bar{N}=\rho \frac{\theta}{2}\left(v_{2}^{2}-v_{1}^{2}\right)=2.7$.

Based on the above, it is now evident that blind cooperation at the peak transmit power is very unlikely to have better NTR compared to the simple point-to-point case! Immediately we deduce that there is a need to decrease the transmit power level, but obviously in a randomized uncoordinated manner.

\section{POWER CONTROL MECHANISM}

The result above suggests that for randomized cooperation to be more efficient, devices need to scale down their transmit power levels. This can be only done in a randomized distributed manner since cooperative devices are unaware of each other. For an arbitrary choice of the pdf of $P_{n}$, it has to be constructed such that the condition in (15) is satisfied. Looking back at (15), it is clear that an IoT device needs to acquire a reasonably accurate estimate $\bar{N}$ in order to achieve acceptable NTR results.

To that end, we have developed a mechanism whereby each device can learn about its own cluster size in an online fashion. The proposed mechanism is built upon the assumption of an orthogonal frequency division multiplexing (OFDM) physical layer (PHY). Considering an OFDM PHY is a trend which has recently picked up and gained traction. For instance, the IEEE 802.15.4g standard group (which is working on developing technology for smart utility networks) includes a provision for an OFDM-based PHY [40]. The adoption of OFDM (also often referred to as multi-carrier modulation) is mainly motivated by its ability to conveniently accommodate

\footnotetext{
${ }^{1}$ We are not aware of practical studies on the expected topologies and network geometries for the IoT. Nevertheless, this is indeed going to be function of the building clutter geometry as well as the typical communication ranges. In any case, the conclusions provided above hold true for a very wide range of values of $\theta$
} 
larger channel bandwidths while featuring less susceptibility to common radio channel impairments [41].

The proposed mechanism designates one OFDM random access symbol (RAS) which contains two sets of subcarriers. The first is a "feed-forward" set, $\mathbf{F}$ whereby devices in cluster $\Omega_{c}$ declare their presence to cooperative devices of hop $c+1$, i.e. $\Omega_{c+1}$. The latter is a "feed-back" set, $\mathbf{B}$ which is used by $\Omega_{c+1}$ to convey the estimated size of $\Omega_{c}$ back to $\Omega_{c}$ itself.

The power control mechanism is further illustrated in Figure 3. At hop $c$, each cooperative device in $\Omega_{c}$ randomly picks one of the $M_{F}$ subcarriers composing the set $\mathbf{F}$ and modulates it with a predefined waveform. Noting that $\left|\Omega_{c}\right|=N^{(c)}$, then $\mathbf{F}$ is represented as

$$
\mathbf{F}=\left\{f_{m}\right\}_{m=1}^{M_{F}}
$$

such that

$$
f_{m} \in\left\{0, \ldots, N^{(c)}\right\}, \sum_{m=1}^{M_{F}} f_{m}=N^{(c)}
$$

With a certain probability, the number of modulated subcarriers (i.e. non-zero elements) in the $\mathbf{F}$ set will be equal to $\left|\Omega_{c}\right|=N^{(c)}$. This is the case when each device picks unique subcarrier. It can be shown that this occurs with a probability of

$$
p_{z}=\left(\frac{M_{F}-N^{(c)}}{M_{F}-N^{(c)}+1}\right)^{N^{(c)}\left(N^{(c)}-1\right) / 2}
$$

Assuming the underlying PHY is based on 128 FFT, and $M_{F}=96$, then the probability $p_{z}$ is greater than $80 \%$ for $N^{(c)} \leq 5$. In other words, for cluster sizes up to $N^{(c)}=5$, it is highly likely that each devices selects a unique RAS subcarrier.

At hop $c+1$, a receiving device will examine the subcarrier set F. It will then apply a threshold test at each of the $M_{F}$ subcarriers. A receiving device obtains an estimate of $N^{(c)}$, denoted here $\widehat{N}^{(c)}$, by simply counting the subcarriers passing the threshold test. It is worthy to note that even without the occurrence of a collision, it is still possible for $\widehat{N}^{(c)} \neq N^{(c)}$ due to fading. The receiving device will then modulate a specific subcarrier within the feedback set, $\mathbf{B}$. The index of that subcarrier is equal to $\widehat{N}^{(c)}$. The same procedure is locally applied by all other cooperative devices in $\Omega_{c+1}$.

The feedback set which composed of $M_{B}$ subcarriers can be represented as:

$$
\mathbf{B}=\left\{b_{m}\right\}_{m=1}^{M_{B}}
$$

such that

$$
b_{m} \in\left\{0, \ldots, N^{(c)}-1, N^{(c)}, N^{(c)}+1 \ldots\right\}
$$

Ideally, the number of zero elements in $\mathbf{B}$ equals $M_{B}-1$. In other words, all $N^{(c+1)}$ devices in $\Omega_{c+1}$ modulate the same subcarrier index (that, which is equal to the estimate $\left.\widehat{N}^{(c)}\right)$. The converse case, i.e. a subset of devices modulating different subcarrier indices is analyzed next. This is related to the inability of devices in $\Omega_{c+1}$ to detect all modulated subcarriers in the set $\mathbf{F}$. One or more devices may fail to detect all subcarriers that have been modulated by devices in $\Omega_{c}$. Subsequently, these less fortunate devices will acquire

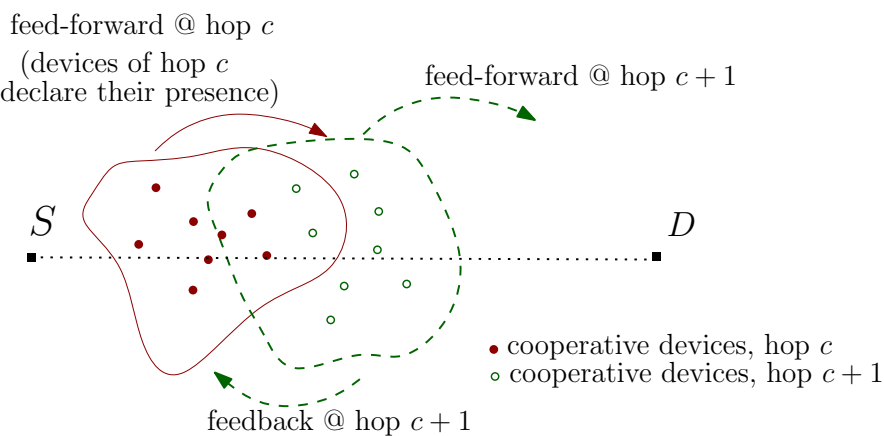

(feedback of number of devices observed, $\widehat{N}^{(c)}$ )

Fig. 3. At hop $c$, devices randomly access the feed-forward portion of the RAS. At hop $c+1$, devices feed-back what they have observed to those of hop $c$.

an estimate $\widehat{N}^{(c)}$ that is smaller than that obtained by the rest of the devices in $\Omega_{c+1}$. The set $\mathbf{B}$ will now contain two (or more) modulated subcarrier indices, contrary to the ideal case of having only one non-zero element. Now, when devices of hop $c$ inspect the set $\mathbf{B}$ they will detect more than one modulated subcarrier. In this case, the maximum likelihood estimate is

$$
\widetilde{N}^{(c)}=\arg \max _{m}\{s(m)\}_{m=1}^{M_{B}}
$$

where $s(m)$ is the signal strength at subcarrier $m$. Assuming that no collisions occur when $\mathbf{F}$ is constructed, then the probability of estimation error, $p_{e}$, is represented by

$$
\begin{aligned}
p_{e} & =\mathbb{P}\left[\left|\widetilde{N}^{(c)}-N^{(c)}\right|>0\right] \\
& =\mathbb{P}\left[\exists m \neq N^{(c)}: s(m)>s\left(N^{(c)}\right)\right]
\end{aligned}
$$

The feedback set $\mathbf{B}$ in the $(c+1)$ th transmission is processed by $\Omega_{c}$. Again, a threshold test is applied to each individual subcarrier. Now, devices in $\Omega_{c}$ will be able to acquire an insight into the size of their own neighborhood. In other words, they obtain an estimate of the size of $\Omega_{c}$, which is $\widetilde{N}^{(c)}$ in (19). For the sake of more clarity, the operation of the scheme is modeled in Figure 4.

So what is this estimate good for? When the next frame $(y+1)$ traverses the network, each device will apply the estimate it obtained for the size of the cooperative cluster, $\widetilde{N}^{(c)}$ to tune the pdf of $P_{n}$. In other words, the random realization of transmit power will be governed by the estimate learned by the device.

\section{Performance ASSESSMent}

In practice, there are few possible sources of error causing $\tilde{N}^{(c)} \neq N^{(c)}$. Two causes have been already highlighted in the previous section, namely: 1) collision on the random access subcarrier set $\mathbf{F}$, and 2) the mismatch between the feed-forward and feedback sets (i.e. $\widehat{N}^{(c)} \neq N^{(c)}$ ) due to radio channel anomalies. Furthermore, the estimate $\widetilde{N}^{(c)}$ which is obtained using frame $y$ is actually used to tune the transmit power of frame $y+1$. Indeed, we expect the channel fading coefficients to be independent from one frame to the next. We recall that 


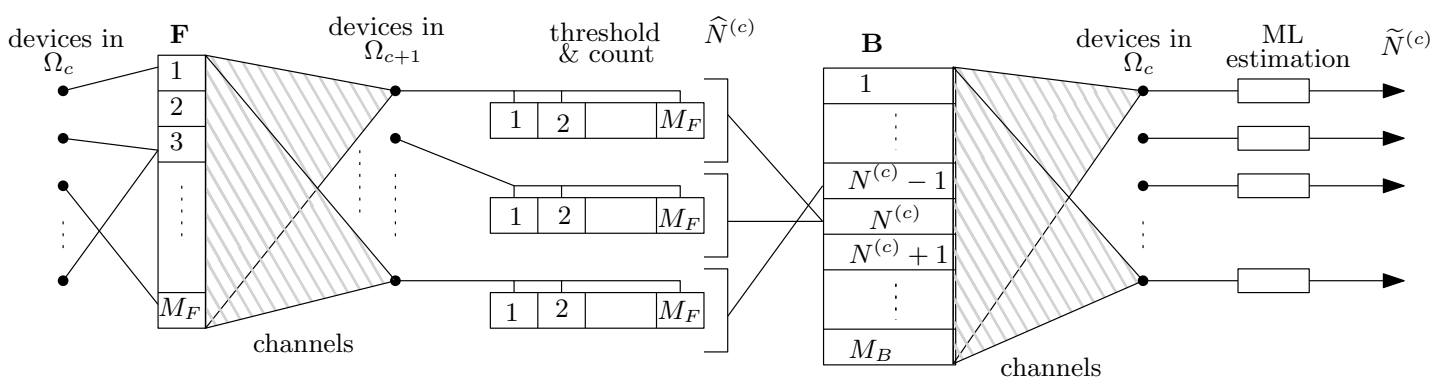

Fig. 4. Model of the power control mechanism.

we assumed a block Rayleigh fading model. Consequently, the channel realization from one frame to the next is independent. We also recall that an IoT setup is expected to employ uncoordinated sleep schedules. Both factors above make us expect that $N^{(c)}(y) \neq N^{(c)}(y+1)$ is generally the norm. As such, even with a perfect estimate $\widetilde{N}^{(c)}$, there is still room for error.

Taking into consideration the various causes of error and mismatch outlined above, we simulated the power control mechanism to assess its performance. In light of Figure 4, a discrete-event system simulation was structured in MATLAB. Each node comprises an independent unit in the discrete-event simulation model of Figure 4. System events include channel realizations, successful link pairs, and assignment of nodes to the feed-forward and feedback sets. Events are updated at a discrete time step equal to one packet duration. Following is a more detailed description:

1) Generate random realization of $N^{(c)}$ based on PPP with mean node intensities of $\bar{N}^{(c)}=2,4,10$.

2) Compute transmit power $\phi$ of the virtual node using an initial value of $P_{n}=P_{\max }$, then compute the corresponding NTR for the first frame, i.e. $y=1$.

3) Generate random realization of $N^{(c+1)}$ nodes such that they lie within the communication range $R$ and offer positive progress towards the origin (where the sink is assumed to be located).

4) The set of devices in $\Omega_{c}$ modulate $\mathbf{F}$, each by randomly selecting a subcarrier in $\mathbf{F}$.

5) After generating random fading channel realizations, devices in $\Omega_{c+1}$ attempt to detect subcarriers in $\mathbf{F}$.

6) Accordingly, they construct the estimate $\widehat{N}^{(c)}$ and then modulate the respective subcarrier index in $\mathbf{B}$.

7) New set of fading channel realizations are generated. Devices in $\Omega_{c}$ attempt to obtain an estimate $\widetilde{N}^{(c)}$ according to (19).

8) Devices in $\Omega_{c}$ now adjust their transmit power levels by generating a random realization according to (15).

9) The steps from 3 to 8 above are repeated for $y=2 \ldots Y$. A uniform pdf was used for the generation of random realizations of the transmit power levels. The simulation results are depicted in Figure 5. We note that the power control mechanism starts to outperform the point-to-point case starting from the second frame, i.e. $y=2$. Up to $5 \mathrm{X}$ improvement is attainable by means of the devised mechanism. The rapid convergence in NTR performance is actually expected. The estimate $\widetilde{N}^{(c)}$ - even if slightly erroneous - quickly positions the transmit power levels in the right range. It can be also observed that the fluctuation from frame to frame increases as $N^{(c)}$ decreases. This is because the error margin (relative to $N^{(c)}$ ) in estimating $\widetilde{N}^{(c)}$ increases and thus its effect on the power adjustment becomes more profound. Such an intuition is reinforced by looking at the normalized standard deviation of NTR versus $\bar{N}$ in Figure 5. Clearly, as $\bar{N}$ increases the results obtained are less likely to fluctuate, i.e. the power control mechanism offers a more stable NTR performance.

\section{CONCLUSIONS}

In this paper, we have made a case for using multihop networking in the context of large-scale IoT. Using blind cooperative transmission in conjunction with multihop networking minimizes underlying protocol overhead, and therefore allows for scalability. We have also shown that large-scale IoT setups tend to be generally noise-limited. Accordingly, we derived an upper bound for the mean transmit power level which must be observed at each device in a cooperative cluster. The bound is derived as function of the size of the cluster, i.e. number of devices. As such, we devised a mechanism for devices to learn the size of their neighborhood without reverting to any exchange of messages with each other; thus maintaining the "blind" property of the system.

The mechanism was assessed in terms of normalized transport rate by means of simulation. The devised mechanism was shown to consistently outperform the simple point-to-point case. Although the convergence of the power mechanism to the desired NTR region is pretty rapid for all device deployment densities, but the performance observed for higher densities was more stable.

\section{REFERENCES}

[1] L. Atzori, A. Iera, and G. Morabito, "The internet of things: A survey," Computer networks, vol. 54, no. 15, pp. 2787-2805, May 2010.

[2] D. Miorandi, S. Sicari, F. De Pellegrini, and I. Chlamtac, "Internet of things: Vision, applications and research challenges," Elsevier Ad Hoc Networks, vol. 10, no. 7, pp. 1497-1516, 2012.

[3] M. Zorzi, A. Gluhak, S. Lange, and A. Bassi, "From today's intranet of things to a future internet of things: a wireless- and mobility-related view," Wireless Communications, IEEE, vol. 17, no. 6, pp. 44-51, December 2010.

[4] C. Drubin, "The internet of things will drive wireless connected devices to. 40.9 billion in 2020.," Microwave Journal, vol. 57(10), no. 51, Aug 2014. 


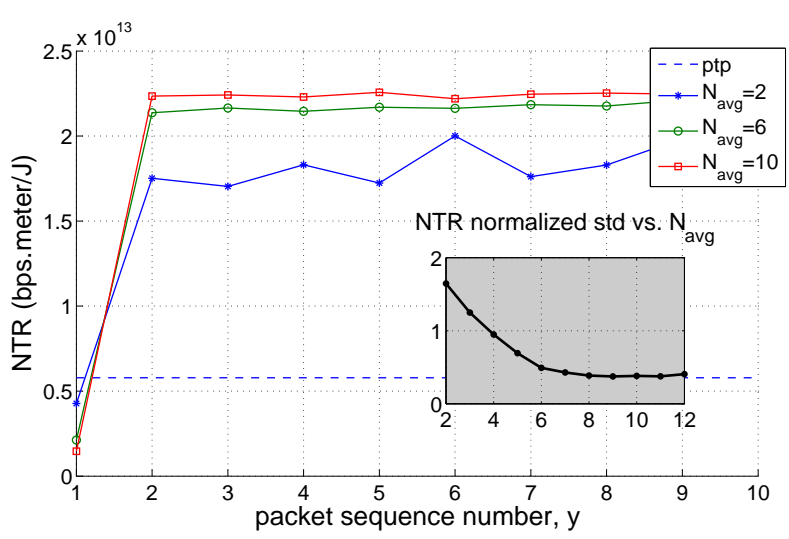

Fig. 5. Simulation results coining the NTR performance for various values of $\bar{N}$ over an ensemble of $Y$ consecutive frames. In the small shaded box, the normalized standard deviation of NTR vs. $\bar{N}$ is displayed. The simulations were carried out for a packet duration of $1 \mathrm{~ms}$, data rate of $1.25 \mathrm{Mbps}$, and a total of 1000 iterations.

[5] IDC, "Internet of things (iot) 2013 to 2020 market analysis: Billions of things, trillions of dollars," Market Analysis Report, 2013.

[6] S. Hodges, S. Taylor, N. Villar, J. Scott, D. Bial, and P.T. Fischer, "Prototyping connected devices for the internet of things," IEEE Computer, vol. 46, no. 2, pp. 26-34, Feb 2013.

[7] A. Markkanen, "Connected versus intelligent devices in the iot and in saunas," The IEEE IoT Newsletter, Mar 102015.

[8] IEEE Standard for Local and Metropolitan Area Networks Part 15.4, "Low-Rate Wireless Personal Area Networks (LR-WPANs) Amendment 3, Physical Layer (PHY) Specifications for Low- Data-Rate, Wireless, Smart Metering Utility Networks," .

[9] "Intelligent transport systems (its); vehicular communications; geonetworking; part 4: Geographical addressing and forwarding for pointto-point and point-to-multipoint communications; sub-part 1: Mediaindependent functionality, v1.2.0," Oct 2013.

[10] "Introducing thread: A new wireless networking protocol for the home," Press Release, Jul 2014.

[11] Yunhao Liu, Yuan He, Mo Li, Jiliang Wang, Kebin Liu, and Xiangyang $\mathrm{Li}$, "Does wireless sensor network scale? a measurement study on greenorbs," Parallel and Distributed Systems, IEEE Transactions on, vol. 24, no. 10, pp. 1983-1993, Oct 2013.

[12] "Request for information, novel methods for information sharing in large scale mobile ad-hoc networks, defense advanced research projects agency (darpa),"

[13] A.A. Abouzeid and N. Bisnik, "Geographic protocol information and capacity deficit in mobile wireless ad hoc networks," Information Theory, IEEE Transactions on, vol. 57, no. 8, pp. 5133-5150, Aug 2011.

[14] Birsen Sirkeci-Mergen and Anna Scaglione, "Randomized space-time coding for distributed cooperative communication," Signal Processing, IEEE Transactions on, vol. 55, no. 10, pp. 5003-5017, 2007.

[15] Yun Li, Zhenghua Zhang, Chonggang Wang, Weiliang Zhao, and HsiaoHwa Chen, "Blind cooperative communications for multihop ad hoc wireless networks," Vehicular Technology, IEEE Transactions on, vol. 62, no. 7, pp. 3110-3122, Sept 2013.

[16] A. Bader, K. Abed-Meraim, and M.-S. Alouini, "A fully distributed georouting scheme for wireless sensor networks," Sensors Journal, IEEE, vol. 13, no. 12, pp. 4585-4586, Dec 2013

[17] M. Sharp, A. Scaglione, and B. Sirkeci-Mergen., "Randomized cooperation in asynchronous dispersive links," IEEE Transactions on Communications, vol. 57, no. 1, pp. 64-68, 2009.

[18] M.S Gokturk, O. Gurbuz, and E. Erkip, "Recomac: A cross-layer cooperative network protocol for wireless ad hoc networks," 5th IEEE International Conference on New Technologies, Mobility and Security (NTMS), Mar 2012.

[19] P. Gupta and P. R. Kumar, "The capacity of wireless networks," IEEE
Transactions on Information Theory, vol. 46, Issue 2, pp. 388-404, March 2000.

[20] J. Andrews, S. Weber, M. Kountouris, and M. Haenggi, "Random access transport capacity," IEEE Transactions on Wireless Communications, vol. 9, no. 6, pp. 2101 - 2111, June 2010.

[21] S. Weber, J.G. Andrews, and N. Jindal, "An overview of the transmission capacity of wireless networks," Communications, IEEE Transactions on, vol. 58, no. 12, pp. 3593-3604, December 2010.

[22] X. Zhang and M. Haenggi, "Random power control in poisson networks," Communications, IEEE Transactions on, vol. 60, no. 9, pp 2602-2611, Sep 2012.

[23] Xinchen Zhang and M. Haenggi, "Delay-optimal power control policies," Wireless Communications, IEEE Transactions on, vol. 11, no. 10, pp. 3518-3527, October 2012.

[24] Tae-Suk Kim and Seong-Lyun Kim, "Random power control in wireless ad hoc networks," Communications Letters, IEEE, vol. 9, no. 12, pp. 1046-1048, Dec 2005.

[25] M. Elmusrati, N. Tarhuni, and R. Jaentti, "Performance analysis of random uniform power allocation for wireless networks in rayleigh fading channels," European Transactions on Telecommunications, vol. 20, pp. 457462, 2009.

[26] S. Vural and E. Ekici, "On multihop distances in wireless sensor networks with random node locations," Mobile Computing, IEEE Transactions on, vol. 9, no. 4, pp. 540-552, April 2010.

[27] R. de Francisco, L. Huang, G. Dolmans, and H. de Groot, "Coexistence of ZigBee Wireless Sensor Networks and Bluetooth inside a Vehicle," IEEE 20th International Symposium on Personal, Indoor and Mobile Radio Communications, 2009,

[28] E. Tanghe, W. Joseph, L. Verloock, and L. Martens, "Evaluation of vehicle penetration loss at wireless communication frequencies," Vehicular Technology, IEEE Transactions on, vol. 57, no. 4, pp. 20362041, July 2008.

[29] R. Hoppe, G. Wlfle, and G.G. Landstorfer, "Measurement of building penetration loss and propagation models for radio transmission into buildings," IEEE VTS 50th Vehicular Technology Conference Fall, vol. 4, pp. 22982302, Sep 1999.

[30] Z. Shelby and C. Borman, The Wireless Embedded Internet, Wiley, 2009.

[31] A. Bader, K. Abed-Meraim, and M.-S. Alouini, "A fully distributed georouting scheme for wireless sensor networks," IEEE Sensors Journal, vol. 13 , no. 12, pp. $4585-4586,2013$.

[32] M. Aljuaid and H. Yanikomeroglu, "On the asymptotic analysis of average interference power generated by a wireless sensor network," IEEE Vehicular Technology Conference (VTC-Fall), 2008.

[33] M. Aljuaid and H. Yanikomeroglu, "A cumulant-based investigation of the impact of secondary users' field size on spectrum-sharing opportunities," IEEE Transactions on Vehicular Technology, vol. 60, no. 7, pp. 3490-3497, 2011.

[34] M. Haenggi, "On routing in random Rayleigh fading networks," IEEE Transactions on Wireless Communications, vol. 4, no. 4, April 2005.

[35] W. Kordecki, "Reliability bounds for multistage structures with independent components.," Statistics and probability letters, vol. 34, no. 1, pp. 43-51, 1997.

[36] C. Sommer, D. Eckhoff, R. German, and F. Dressler, "A computationally inexpensive empirical model of ieee $802.11 \mathrm{p}$ radio shadowing in urban environments," in Wireless On-Demand Network Systems and Services (WONS), 2011 Eighth International Conference on, Jan 2011, pp. 84-90.

[37] T. Rappaport, Wireless Communications: Priciples and Practice, Prentice Hall, 2nd edition, 2001.

[38] A. Patel, J. Aparicio, N. Tas, M. Loiacono, and J. Rosca, "Assessing communications technology options for smart grid applications," in proceedings of IEEE International Conference on Smart Grid Communications (SmartGridComm), 2011.

[39] E. S. Sousa and J. A. Silvester, "Optimum Transmission Ranges in a Direct-Sequence Spread-Spectrum Multihop Packet Radio Network," IEEE Journal on Selected Areas in Communications, vol. 8, Issue 5, pp. 762-771, June 1990.

[40] E. Monnerie, J. Buffington, S. Shimada, and K. Waheed, "IEEE 802.15.4g OFDM PHY Overview," doc. IEEE 802.11-10/1305r1, January 2011.

[41] H. Schulze and C. Lueders, Theory and Applications of OFDM and CDMA, John Wiley and Sons Ltd, 1st edition, 2005. 